\title{
MOLECULAR CLOUD CORE AND CS OUTFLOW ASSOCIATED WITH HARO 4-255 FIR
}

\author{
K. Tatematsu, ${ }^{1,2}{ }^{2}$ T. Umemoto, ${ }^{1}$ Y. Murata,${ }^{3}$ H. Chen, ${ }^{4}$ N. Hirano, 5 \\ and H. TAKABA 6 \\ 1 Nobeyama Radio Observatory, Minamisaku, Nobeyama, Nagano 384-13, Japan \\ 2 Present address: Dept. of Astronomy, University of Texas, Austin, TX 78712, USA \\ 3 Institute of Space and Astronautical Sci., Yoshinodai, Sagamihara, Kanagawa 229, Japan \\ 4 Institute for Astronomy, University of Hawaii, Honolulu, HI 96822, USA \\ 5 Lab. of Astronomy \& Geophys., Hitotsubashi University, Kunitachi, Tokyo 186, Japan \\ 6 Communications Research Lab., Kashima, Ibaraki 314, Japan
}

\begin{abstract}
The region containing Haro 4-255 FIR is studied in CS (1-0) with the $45 \mathrm{~m}$ telescope and the Nobeyama Millimeter Array. We have detected intense CS outflow. The CS abundance is found to be enhanced in the outflow lobe. The aperture synthesis data show two groups of emission, one of which is likely to impede the blueshifted CS lobe.
\end{abstract}

\section{INTRODUCTION}

We carried out the systematic survey of molecular cloud cores in the Orion A GMC, and catalogued 125 cores (Tatematsu et al. 1993). In this paper, we select one core toward Haro 4-255 FIR for a further study. Haro 4-255 FIR is a far-infrared object Evans et al. (1986) discovered, and is located $45^{\mathrm{m}} \mathrm{NE}$ of the classical T Tauri star Haro 4-255. Recently, Anglada et al. (1992) detected two radio continuum sources at $3.6 \mathrm{~cm}$ with VLA in this region.

\section{OBSERVATION}

Single-dish CS (1-0) data were obtained with the Nobeyama $45 \mathrm{~m}$ telescope (HPBW $=36^{\prime \prime}$ at 49 GHż). Details of the observation are given in Tatematsu et al. (1993). Aperture-synthesis CS (1-0) data were obtained with the Nobeyama Millimeter Array (NMA). Two fields were observed. The synthesized beamwidth was $15^{n} \times 12^{\prime \prime}\left(\right.$ P. A. $=-17^{\circ}$ ).

\section{RESULTS}

Figure 1 shows a CS position-velocity diagram passing through one of the VLA sources. Broad emission is observed around this VLA source, and most likely represents a CS outflow. Assuming that the momentum of the CS outflow is comparable with that of the previously known CO outflow (Levreault 1988; Morgan et al. 1991), we estimate the CS abundance to be about $10^{-8}$, which is one order of magnitude larger than the value in the molecular cloud cores. From the same assumption, the total mass of the molecular outflow might be three times as large as the previous estimate solely based on the $\mathrm{CO}$ data. The aperture-synthesis observation detected two features of emission, which roughly coincide with local peaks within the core on the singledish map (Fig. 2). One of them is located between Haro 4-255 FIR and the T Tauri star, and its 
distribution is very similar to that in $\mathrm{HCO}^{+}$by Chen et al. (1992). No emission from the CS outflow was detected in the aperture-synthesis observation. The blueshifted wing of the CS outflow is weak (Fig. 1) probably because the densest part of the core, which is seen as the above aperture-synthesis feature, impedes the CS outflow. The total mass detected in the aperturesynthesis observation is $17 M_{\odot}$, which is $5 \%$ of the mass observed in the single-dish observation.

\section{REFERENCES}

Anglada, G., Rodriguez, L., Cant6, J., Estalella, R., \& Torrelles, J. M. 1992, ApJ, 395, 494

Chen, H., Fukui, Y., \& Yang, J. 1992, ApJ, 398, 544

Evans, N. J., II., Levreault, R. M., \& Harvey, P. M. 1986, ApJ, 301, 894

Levreault, R. M. 1988, ApJS, 67, 283

Morgan, J. A., Schloerb, F. P., Snell, R. L., \& Bally, J. 1991, ApJ, 376, 618

Tatematsu, K., et al. 1993, ApJ, in press

FIG. 1 (Right) - Single-dish CS $(1-0)$ positionvelocity diagram passing through one VLA source with a position angle of $-45^{\circ}$. The lowest contour level is $0.68 \mathrm{~K} T_{A}{ }^{*}$ and the level interval is $0.34 \mathrm{~K}$ $T_{A}{ }^{*}$.

FIG. 2 (Bottom) - Comparison of the single-dish CS map [2.16 and $4.32 \mathrm{~K} \mathrm{~km} \mathrm{~s}^{-1}$ in $T_{A}{ }^{*}$ ], aperture-synthesis CS map $[(-2,2,3,4,5) \times 1.77 \mathrm{~K}$ $\mathrm{km} \mathrm{s}^{-1}$ in $T_{b}$ ], and the lobes of the CO outflow [4.35 $\mathrm{K} \mathrm{km} \mathrm{s}^{-1}$, Morgan et al. 1991].

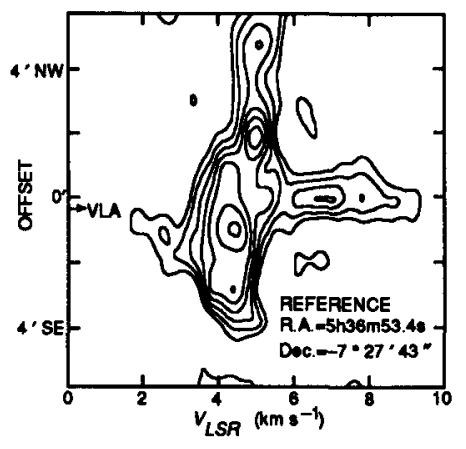
T Tauri star.

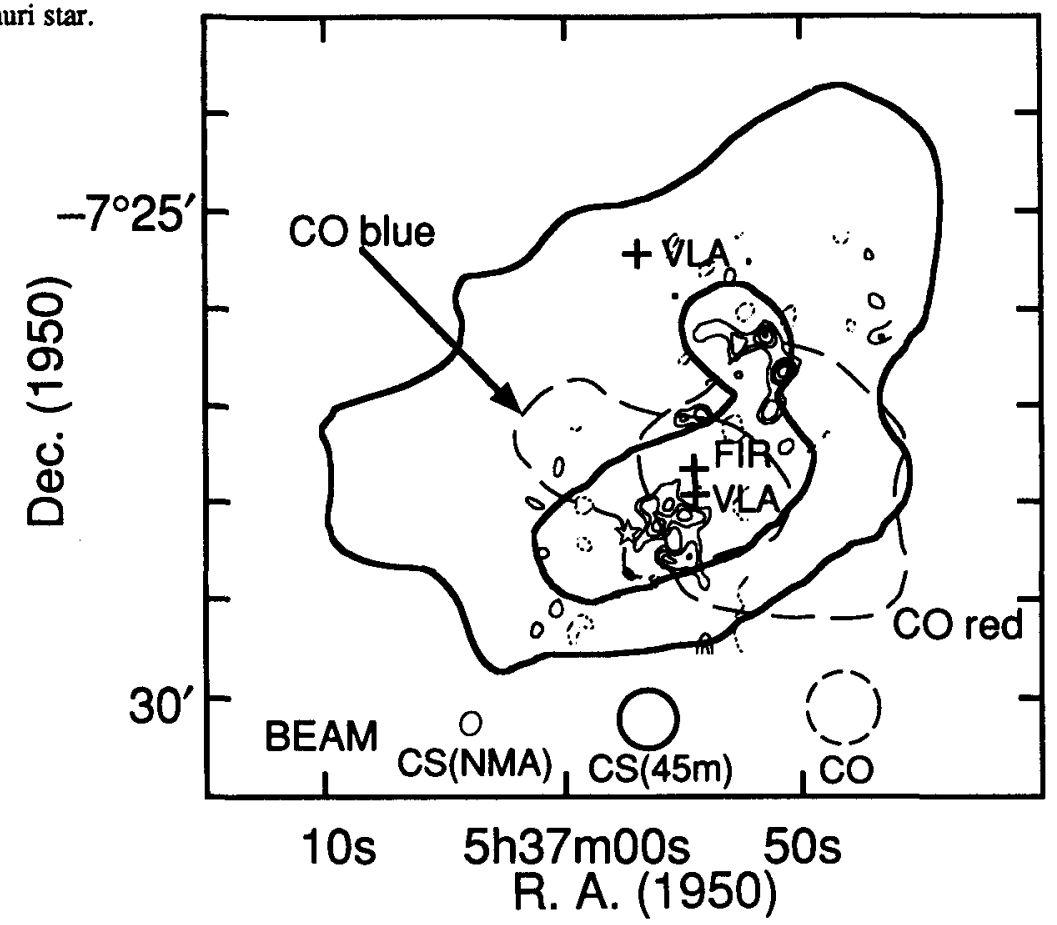

